\title{
Cardioprotective Plant Extracts
}

\author{
Sankarganash Arunachaalam, Umapriya, Selvaraj Kunjiappan
}

\begin{abstract}
Cardiovascular disease is assumingas a major cause of morbidity and mortality worldwide. In traditional Indian medicinal system, many plants were used for thetreatment of cardiac failure. Medicinalplants are used in various ailments due to efficacy, low cost, ease availability and safety.Because of these advantages the usage ofmedicinal plant increased by the medicinal practitioners in their day to day practice. Inthis review article, we discuss about the plant extracts from various part of the plantwhich is used to cure or protect against the cardiomyopathy induced by many inducingfactors.
\end{abstract}

Keywords: Plant extract, Medicinal Plant, Cardiovascular disease.

\section{INTRODUCTION}

Cardiovascular disorder refers as the diseases in related to heart and blood vessels. The most common ones are the heart muscle, strokes, heart attacks, heart failure and those caused by high blood pressure. In Worldwide, cardiovascular disease is assuming as a major cause of morbidity and mortality [1]. Approximately 16.7 live per annum were estimated in worldwide by WHO report 2003. In 2020 it may be raised to $36.3 \%$ [2]. Moreover, the cardiovascular diseases expected to jump inranking from fourth to first, due to premature death and disability [3]. The predisposing factors to cardiovascular diseases include cigarette smoking, elevated cholesterol, hypertension, obesity, physical inactivity and diabetes.

In traditional Indian medicinal system, many plants were used for the cardioprotection [4]. Because it does not produce any harmful effect and does not cause any side effects. The discovery of many important modern drugs has the starting point as natural products. ThePotential source of drugs as a medicinalplant because there were rich in secondary metabolites and essential oil of therapeutic medicines [5]. Medicinal plants were used in various ailments are due to being economical, effective, their ease availability and due to their safety [5]. Because of these advantages the usage of medicinal plant were increased by the medicinal practitioners in their day to day practice [6]. defence and for other biological functions however, foods obtained by plants contain a wide range of non- nutrient phytochemicals [7]. From the ancient days, plants were used

Revised Manuscript Received on December 15, 2019.

Dr. Sankarganash Arunachaalam*, Department of Biotechnology, School of Bio and Chemical Engineering, Kalasalingam Academy of Research and Education, Krishnankoil, Virudhunagar Dt., Tamilnadu PIN 626126, India. Email: sankarganesh@klu.ac.in

Umapriya M., Department of Biotechnology, School of Bio and Chemical Engineering, Kalasalingam Academy of Research and Education, Krishnankoil, Virudhunagar Dt., Tamilnadu PIN 626126, India. Email:

Selvaraj Kunjiappan, Department of Biotechnology, School of Bio and Chemical Engineering, Kalasalingam Academy of Research and Education, Krishnankoil, Virudhunagar Dt., Tamilnadu PIN 626126, India. Email: selvarj@klu.ac.in
Phytochemicals that are synthesized by plants for their umapriya.m@klu.ac.in

for the medicinal purposes as a therapy against diseases and ailments. The Plants contain

phytochemicals, they act as an antioxidant and they supply necessary nutrients, for various function of the human body and it reduce the occurrence of many diseases. The plant parts (herbs, leaves, stem, root, etc.,) also have a medicinal values, used to prevent alleviate or cure several human diseases [8]. We ingest these plant foods to meet our nutritional needs; we also ingest a wide variety of these non-nutrient

phytochemicals. These phytochemicals have the potential for preventing chronic diseases and also non-toxic [7]. Today, the world consumes plants as a traditional medicine. In developing countries, the traditional medicine is directed to the socio-economic status and well being of the rural communities [9]. This has led to the increasing search for plants with medicinaluse.

The "Magic Bullet Theory" suggests that the only pure compounds are the most efficacious. So the emerging new field of nutrigenomics and pharmacogenomics will play an important role in determining the interaction of these complex substances with the genetic variability of individuals and will determine the individual response and its magnitude to phytochemicals [10]. In this review article, we are discussing about the plant extract from various part of the plant which is used to cure or protect against the cardiomyopathy induced by many inducing factors.

\section{CARDOMYOPATHY INDUCING FACTORS}

Cardiomyopathy is a disease which was refers to the heart related diseases. It was induced by different inducing factors. Some of as follow as

\section{A. Carbon Tetrachloride-Induced Cardiotoxicity (CCl4)}

CCl4 is a renowned model compound. It is used to producing chemical toxicity by creation of free radicals in liver, kidney, heart, lung, testis, brain and blood. After the intake of CCL4, the liver was transformed by cytochrome P450 which could leads to the formation of trichloromethyl free radicals. Because of this lipids modication, is to form of peroxidation of polyunsaturated fatty acids in the cell membrane. For protection against cardiomyopathy, the most important is antioxidant activity or the inhibition of the generation of free of radicals [11].

\section{B. Diabetics induced and cardiomyopathy}

Diabetes is one of the alarming diseases in the developing and developed world. It affects a number of organ systems including kidneys, liver, eyes, reproductive system, heart etc. Diabetic cardiomyopathy is one of the leading causes of death compared to other complications of diabetes.

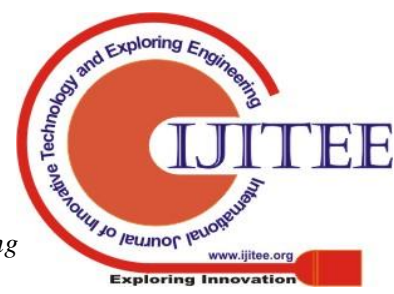




\section{Cardioprotective Plant Extracts}

The oxidative stress is a key factor in etiology of diabetic complications like cardiomyopathy and nephropathy. Theincidence of diabetic heart failure is correlated with increase in age, blood pressure, weight and cholesterol levels. It is characterized by a series of alterations in structure and functions of the heart, without a exclusion of references, it should be less than 5\%. coronary artery disease and hypertension, ultimately leading to heart failure. Pathophysiology includes left ventricular hypertrophy, systolic and diastolic dysfunctions.

C. Isoproterenol induced myocardial Infarction Isoproterenol (ISO), a synthetic adrenoceptor agonist. It has been found to induce myocardial injury in rat as a result of disturbance in physiological balance between production of free radicals and antioxidative defense system [12].

\section{Doxorubicin Induced Cardiomyopathy}

Doxorubicin (DOX) is an anticancer drug which belongs to anthracycline antibiotics. It is being used widely for treatment of various types of tumor malignancies. But in the clinical use, it causes serious and undesirable side effects especially dose-dependent myocardial injury, leads to heart attack [13]. DOX induced cardiomyopathy by free radical generation and mitochondrial dysfunction iron-dependent oxidative damage of biological macromolecules, and protein oxidation [14].

\section{E. Streptozotocin induced cardiotoxicity}

Streptozotocin is a naturally occurring chemical. It is toxic to the insulin-producing beta cells of the pancreas in mammals. In medical research, it is used to produce Type 1 diabetes in a large dose as well as Type 2 diabetes with multiple low doses in test animal. It is a

glucosaminenitrosourea compound and it is toxic to cells by causing damage to the DNA. The activation of poly ADP-ribosylation, induced by DNA damage which leads to the formation of diabetes induction than DNA damages itself [15].

\section{F. Catecholamine Induced Myocardial}

In the cellular mechanism of cardiomyopathy, catecholamines which are highly oxidative metabolites lead lipid peroxidation. Catecholamines accelerates rate of peroxidation in membrane phospholipids and releases free fatty acids into plasma. Because of this the phospholipase A2, catecholamines-induced hyperlipidemia [16].

\section{G. Isoprenaline-induced cardiotoxicity}

Isoprenaline is a syntheticcatecholamine and b adrenergic agonist known to cause oxidative damage andcardiac dysfunction in rats. It is considered as the most authenticated model for theevaluation of drugs in Cardiomyopathy (Mohanty et al., 2004).

\section{H. Plant Extract}

The fresh leaves/fruits/ root/ tubers/ bark/ herb/ peels/ seeds/ pollen grains or whole plant were extracted with ethanol or methanol or aqueous or hydrochloric acid or alcohol were used to determine the cardioprotective activity.

Table I: 1 Extracts Of Roots \&Tubers For Cardiomyopathy Activity

\begin{tabular}{|c|c|c|c|c|c|c|}
\hline S.No & Plant Name & $\begin{array}{c}\text { Parts } \\
\text { of } \\
\text { Plant }\end{array}$ & Extract & $\begin{array}{c}\text { CMP } \\
\text { Induction }\end{array}$ & Concentartion & Reference \\
\hline 1 & $\begin{array}{c}\text { Diosgenin } \\
\text { (Dioscorea } \\
\text { opposita })\end{array}$ & Tuber & Aqueous & Doxorubicin & & $\begin{array}{c}\text { Chih-Tai Chen } \text { et al., } \\
2015\end{array}$ \\
\hline 2 & $\begin{array}{c}\text { Daucus carota } \\
\text { Linn. }\end{array}$ & $\begin{array}{c}\text { Tuber } \\
\text { ous } \\
\text { Root }\end{array}$ & Aqueous & Isoproterenol & 250 and 500mg & $\begin{array}{c}\text { P.Muralidharan } \text { et al } \\
2008\end{array}$ \\
\hline
\end{tabular}

Table II. Extract of Leaves/Leaf for cardiomyopaty activity

\begin{tabular}{|c|c|c|c|c|c|c|}
\hline S. No & Plant Name & $\begin{array}{c}\text { Parts } \\
\text { Of } \\
\text { Plant }\end{array}$ & Extract & $\begin{array}{c}\text { CMP } \\
\text { Induction }\end{array}$ & Concentration & Reference \\
\hline 1 & Euphorbia hirta & Leaves & Ethanol & Normal & & $\begin{array}{c}\text { Md Reyad-Ul- } \\
\text { Ferdous } \text { et al., } \\
2017\end{array}$ \\
\hline 2 & Costus Afer & Leaf & Methanol & $\begin{array}{c}\text { Carbon } \\
\text { Tetrachloride }\end{array}$ & $\begin{array}{c}100,200,300 \\
\text { and } 400 \mathrm{mg}\end{array}$ & $\begin{array}{c}\text { U. O. Njoku } \text { et al., } \\
2017\end{array}$ \\
\hline 3 & $\begin{array}{c}\text { Andrographis } \\
\text { paniculata }\end{array}$ & Leaves & Methanol & isoproterenol & $200 \mathrm{mg}$ & $\begin{array}{c}\text { Dipendra Kumar Sah } \\
\text { and } \\
\text { Nagarathana }\end{array}$ \\
\hline
\end{tabular}




\begin{tabular}{|c|c|c|c|c|c|c|}
\hline & & & & & & $\begin{array}{c}\text { P.K.M. } \\
2016\end{array}$ \\
\hline 4 & $\begin{array}{c}\text { Abroma augusta } \\
\text { L. }\end{array}$ & Leaves & Aqueous & Diabetics & $\begin{array}{l}100 \mathrm{mg} \text { and } \\
200 \mathrm{mg}\end{array}$ & $\begin{array}{l}\text { Ritu Khanra et al., } \\
2016\end{array}$ \\
\hline 5 & Cassia alata (L.) & Leaves & Methanol & Doxorubicin & $\begin{array}{l}100 \mathrm{mg} 200 \mathrm{mg} \\
\text { and } 400 \mathrm{mg}\end{array}$ & $\begin{array}{c}\text { Vishnu Neharkar } \\
\text { et al., } 2016\end{array}$ \\
\hline 6 & Ipomoea batatas & Leaf & Ethanol & Doxorubicin & $\begin{array}{c}600 \mathrm{mg} \text { and } \\
900 \mathrm{mg}\end{array}$ & $\begin{array}{c}\text { Balakrishna } \\
\text { Somashekar et al., } \\
2015\end{array}$ \\
\hline 8 & $\begin{array}{c}\text { Newbouldia } \\
\text { laevis }\end{array}$ & $\begin{array}{l}\text { Leaf } \\
\text { and } \\
\text { Root }\end{array}$ & Water & $\begin{array}{c}\text { Carbon } \\
\text { Tetrachloride }\end{array}$ & $800 \mathrm{mg}$ & $\begin{array}{c}\text { K. N. Agbafor et al., } \\
2015\end{array}$ \\
\hline 9 & $\begin{array}{r}\text { Moringa } \\
\text { Oleifera }\end{array}$ & Leaf & Ethanol & Doxorubicin & $\begin{array}{c}500 \mathrm{mg} \text { and } \\
1000 \mathrm{mg}\end{array}$ & $\begin{array}{l}\text { Fikriansyah et al., } \\
2015\end{array}$ \\
\hline 10 & Carissa opaca & Leaves & Water & $\mathrm{CCl} 4$ & $\begin{array}{c}50 \mathrm{mg} \text { and } \\
200 \mathrm{mg}\end{array}$ & $\begin{array}{c}\text { Sumaira Sahreen } \\
\text { et al., } 2014\end{array}$ \\
\hline 11 & $\begin{array}{c}\text { Parkia } \\
\text { Biglobosa }\end{array}$ & Leaf & Methanol & Doxorubicin & $50-75 \mathrm{mg}$ & $\begin{array}{l}\text { Komolafe K et al., } \\
2013\end{array}$ \\
\hline 12 & $\begin{array}{c}\text { Ocimum } \\
\text { basilicum L. }\end{array}$ & Leaves & Ethanol & Isopreterenol & $\begin{array}{c}10,20 \text { and } \\
40 \mathrm{mg}\end{array}$ & $\begin{array}{c}\text { Fatemeh } \\
\text { Fathiazad } \text { et al., } 2012\end{array}$ \\
\hline 13 & $\begin{array}{c}\text { Parkia } \\
\text { Biglobosa }\end{array}$ & Leaf & Methanol & Doxorubicin & $\begin{array}{c}25,75 \text { and } \\
100 \mathrm{mg}\end{array}$ & $\begin{array}{c}\text { Komolafe K et al., } \\
2013\end{array}$ \\
\hline 14 & Aegle marmelos & Leaf & Ethanol & Alloxan & $200 \mathrm{mg}$ & $\begin{array}{c}\text { Rajbir Bhatti et } \\
\text { al., } 2011\end{array}$ \\
\hline 15 & $\begin{array}{c}\text { Spinacia } \\
\text { oleracea Linn. }\end{array}$ & Leaf & Methanol & isoproterenol & 100 and $200 \mathrm{mg}$ & $\begin{array}{c}\text { Shivaranjani } \\
\text { Vutharadhi, } \text { et al., } \\
2016\end{array}$ \\
\hline 16 & Viscum album L. & Leaf & Methanol & Normal & & $\begin{array}{c}\text { Eylem Suveren et } \\
\text { al., } 2017\end{array}$ \\
\hline
\end{tabular}

Table III: Extract of Fruits for cardiomyopathy activity

\begin{tabular}{|c|c|c|c|c|c|c|}
\hline S. No & Plant Name & $\begin{array}{c}\text { Parts } \\
\text { Of } \\
\text { Plant }\end{array}$ & Extract & $\begin{array}{c}\text { CMP } \\
\text { Induction }\end{array}$ & Concentration & Reference \\
\hline 1 & $\begin{array}{c}\text { Parmentiera } \\
\text { cereifera Seem }\end{array}$ & Fruits & Methanol & Normal & $500 \mathrm{mg}$ & $\begin{array}{c}\text { Md. Reyad-ul-Ferdous } \\
\text { et al., 2015 }\end{array}$ \\
\hline 2 & $\begin{array}{c}\text { Pithecellobium } \\
\text { Dulce }\end{array}$ & $\begin{array}{c}\text { Fruit } \\
\text { Peel }\end{array}$ & Ehanol & isoproterenol & $200 \mathrm{mg}$ & $\begin{array}{c}\text { PakutharivuThangarajan } \\
\text { et al., 2014 }\end{array}$ \\
\hline 3 & Garcinia indica & Fruit & Ethanol & Isoproterenol & $\begin{array}{c}400 \mathrm{mg} \text { and } \\
800 \mathrm{mg}\end{array}$ & $\begin{array}{c}\text { Vandana Panda et al., } \\
2014\end{array}$ \\
\hline 4 & $\begin{array}{c}\text { Momordica Dioca } \\
\text { Roxb. }\end{array}$ & Fruit & Methanol & Doxorubicin & & $\begin{array}{c}\text { S. Shamala and K.L. } \\
\text { Krishna 2013 }\end{array}$ \\
\hline 5 & $\begin{array}{c}\text { Andrographis } \\
\text { paniculata }\end{array}$ & Leaves & Methanol & Isoproterenol & $200 \mathrm{mg}$ & $\begin{array}{c}\text { Dipendra Kumar Sah } \\
\text { and Nagarathana }\end{array}$ \\
& \multicolumn{2}{|c|}{} & & & & P.K.M. 2016 \\
\hline
\end{tabular}




\section{Cardioprotective Plant Extracts}

\begin{tabular}{|c|c|l|c|c|c|c|}
\hline 6 & Cassia alata (L.) & Leaves & Methanol & Doxorubicin & $\begin{array}{c}100 \mathrm{mg}, 200 \mathrm{mg} \\
\text { and 400mg }\end{array}$ & $\begin{array}{r}\text { Vishnu Neharkar } \text { et al., } \\
2016\end{array}$ \\
\hline 5 & $\begin{array}{r}\text { Vaccinium } \\
\text { meridionale }\end{array}$ & Fruit & NonAlcholic & & & $\begin{array}{c}\text { Yasmin E. Lopera } \text { et al., } \\
2013\end{array}$ \\
\hline 6 & Punica granatum L. & Fruit & Water & Doxorubicin & $100 \mathrm{mg}$ & $\begin{array}{c}\text { Mohammad Hassanpour } \\
\text { Fard } \text { et al., 2011 }\end{array}$ \\
\hline 7 & Aristotelia chilensis & Fruits & Phenolic & Normal & & $\begin{array}{r}\text { Carlos L. Ce'spedes } \text { et } \\
\text { al., 2008 }\end{array}$ \\
\hline 8 & Zingiber officinale & Fruit & Ethanol & Diabetics & $50 \mathrm{mg}$ & $\begin{array}{c}\text { Behrouz Ilkhanizadeh } \text { et } \\
\text { al., 2016. }\end{array}$ \\
\hline 9 & Citrus macroptera & Fruit & Ethanol & Isoproterenol & $500 \mathrm{mg}$ & Sudip Paul et al., 2017 \\
\hline 10 & PhoenixdactyliferaL. & Pulp & Aqueous & Isoproterenol & 250 and & $\begin{array}{c}\text { MohammedAl-Yahya } \text { et } \\
\text { al., 2016 }\end{array}$ \\
\hline
\end{tabular}

Table IV: Extract of Seed for cardiomyopathy activity

\begin{tabular}{|c|c|c|c|c|c|c|}
\hline S. No & Plant Name & $\begin{array}{c}\text { Parts } \\
\text { of Plant }\end{array}$ & Extract & $\begin{array}{c}\text { CMP } \\
\text { Induction }\end{array}$ & Concentration & Reference \\
\hline 1 & $\begin{array}{c}\text { Caesalpinia } \\
\text { crista } \text { Linn. }\end{array}$ & Seed & $\begin{array}{c}\text { Achol \& } \\
\text { Aqueous }\end{array}$ & Isoproterenol & $400 \mathrm{mg}$ & $\begin{array}{c}\text { Sharma Rajesh } \\
\text { Kumar, Sharma } \\
\text { Ashish Kumar } \\
2013\end{array}$ \\
\hline 2 & $\begin{array}{c}\text { Grapeseed } \\
\text { Proanthocyanidin }\end{array}$ & Seed & Water & Doxorubicin & $200 \mathrm{mg}$ & $\begin{array}{c}\text { M. Abirami and U. } \\
\text { Kanagavalli 2013 }\end{array}$ \\
\hline
\end{tabular}

Table V: Extract of Flower for cardiomyopathy activity

\begin{tabular}{|c|c|c|c|c|c|c|}
\hline S. No & Plant Name & $\begin{array}{c}\text { Parts } \\
\text { Of } \\
\text { Plant }\end{array}$ & Extract & $\begin{array}{c}\text { CMP } \\
\text { Induction }\end{array}$ & Concentration & Reference \\
\hline 1 & Marigold & Flower & Hydrocholic & Diabetics & $\begin{array}{c}250 \text { and } \\
500 \mathrm{mg}\end{array}$ & $\begin{array}{c}\text { Esmaeel } \\
\text { Ebahimi } \text { et al., } \\
2016\end{array}$ \\
\hline 2 & Tecoma stans & Flower & Ethanol & isoproterenol & $200 \mathrm{mg}$ & $\begin{array}{c}\text { Shanmukha } \\
\text { Ittagi } \text { et al., } \\
2014\end{array}$ \\
\hline 3 & Tecoma stans & Flower & Ethanol & Streptozotocin & $120 \mathrm{mg}$ & $\begin{array}{c}\text { Kameshwaran } \\
\text { Sugavanam } \text { et } \\
\text { al., } 2013\end{array}$ \\
\hline
\end{tabular}

Table VI: Extract of Bark for cardiomyopathy activity

\begin{tabular}{|c|c|c|c|c|c|c|}
\hline S. No & Plant Name & $\begin{array}{c}\text { Parts } \\
\text { Of } \\
\text { Plant }\end{array}$ & Extract & $\begin{array}{c}\text { CMP } \\
\text { Induction }\end{array}$ & Concentration & Reference \\
\hline 1 & $\begin{array}{c}\text { Terminalia } \\
\text { Paniculata Roth }\end{array}$ & Bark & Ethanol & Doxorubicin & $\begin{array}{c}200 \mathrm{mg} \text { and } \\
400 \mathrm{mg}\end{array}$ & $\begin{array}{c}\text { Davey., M.S and } \\
\text { Atlle., C.V } \\
2011\end{array}$ \\
\hline
\end{tabular}




\begin{tabular}{|c|c|c|c|c|c|c|}
\hline 2 & Cassia Fistula & Bark & Methanol & Normal & $400 \mathrm{mg}$ & $\begin{array}{c}\text { Khatib N. A. } \\
2010\end{array}$ \\
\hline 3 & $\begin{array}{c}\text { Terminalia } \\
\text { arjuna }\end{array}$ & Bark & Aqueous & Normal & $750 \mathrm{mg}$ & $\begin{array}{c}\text { Subir K Maulik } \\
\text { et al., 2016 }\end{array}$ \\
\hline
\end{tabular}

Table VII: Extract of Herb for cardiomyopathy activity

\begin{tabular}{|c|c|c|c|c|c|c|}
\hline S. No & Plant Name & $\begin{array}{c}\text { Parts } \\
\text { Of } \\
\text { Plant }\end{array}$ & Extract & $\begin{array}{c}\text { CMP } \\
\text { Induction }\end{array}$ & Concentration & Reference \\
\hline 1 & $\begin{array}{c}\text { Solanum } \\
\text { surettense }\end{array}$ & Herb & Ethanol & Isoprotenol & $\begin{array}{r}200 \text { and } \\
400 \mathrm{mg}\end{array}$ & $\begin{array}{c}\text { Chitikela P } \\
\text { Pullaiah } \text { et al., } \\
2015\end{array}$ \\
\hline 2 & Bacopa monnieri & Herb & Ethanol & Normal & $30,100 \mu \mathrm{g} / \mathrm{ml}$ & $\begin{array}{c}\text { Sirintorn } \\
\text { Srimachai } \text { et al., } \\
2016 .\end{array}$ \\
\hline 3 & $\begin{array}{c}\text { Nepeta } \\
\text { deflersiana }\end{array}$ & Herb & Ethanol & isoproterenol & $2000 \mathrm{mg}$ & $\begin{array}{c}\text { Areej } \\
\text { Mohammad } \\
\text { Al-Taweela } \text { et } \\
\end{array}$ \\
& & & & & & \\
al., 2017
\end{tabular}

Table VIII: Extract of Whole Plant for cardiomyopathy activity

\begin{tabular}{|c|c|c|c|c|c|c|}
\hline S. No & Plant Name & $\begin{array}{c}\text { Parts } \\
\text { Of } \\
\text { Plant }\end{array}$ & Extract & $\begin{array}{c}\text { CMP } \\
\text { Induction }\end{array}$ & Concentration & Reference \\
\hline 1 & $\begin{array}{l}\text { Green Combination } \\
\text { Terminalia arjuna } \\
\text { and Piper nigrum }\end{array}$ & $\begin{array}{l}\text { Whole } \\
\text { Plant }\end{array}$ & Aqueous & Catecholamine & $100 \mathrm{mg}$ & $\begin{array}{c}\text { Fatiqa Zafar } e t \\
\text { al., } 2015\end{array}$ \\
\hline 2 & $\begin{array}{c}\text { Sida Rhombifolia } \\
\text { Linn. }\end{array}$ & $\begin{array}{r}\text { Whole } \\
\text { Plant }\end{array}$ & Ethanol & Isoproterenol & $\begin{array}{l}100 \mathrm{mg} \text { and } \\
200 \mathrm{mg}\end{array}$ & $\begin{array}{c}\text { Ramadoss S et } \\
\text { al., } 2012\end{array}$ \\
\hline 3 & $\begin{array}{c}\text { Evolvulus } \\
\text { Alsinoides Linn }\end{array}$ & $\begin{array}{l}\text { Whole } \\
\text { Plant }\end{array}$ & Methanol & Isoproterenol & $\begin{array}{l}100 \mathrm{mg} \text { and } \\
200 \mathrm{mg}\end{array}$ & $\begin{array}{c}\text { Sudhakumari et } \\
\text { al., } 2012\end{array}$ \\
\hline 4 & $\begin{array}{c}\text { Aerva lanata } \\
\text { (Linn.) }\end{array}$ & $\begin{array}{l}\text { Whole } \\
\text { Plant }\end{array}$ & $\begin{array}{c}\text { Aqueous \& } \\
\text { Ethanol }\end{array}$ & Doxorubicin & $200 \mathrm{mg}$ & $\begin{array}{c}\text { Paramasivam } \\
\text { Ragavendran et } \\
\text { al., } 2012\end{array}$ \\
\hline 5 & $\begin{array}{l}\text { Commiphora } \\
\text { Mukul }\end{array}$ & $\begin{array}{r}\text { Whole } \\
\text { Plant }\end{array}$ & $\mathrm{HCL}$ & Isoprenaline & $\begin{array}{l}100,200 \text { and } \\
400 \mathrm{mg}\end{array}$ & $\begin{array}{c}\text { Shreesh Ojha et } \\
\text { al., } 2011\end{array}$ \\
\hline 6 & $\begin{array}{c}\text { Hybanthus } \\
\text { Enneaspermus } \\
\text { (Linn.) }\end{array}$ & $\begin{array}{l}\text { Whole } \\
\text { Plant }\end{array}$ & Aqueous & Isoproterenol & $500 \mathrm{mg}$ & $\begin{array}{c}\text { Radhika S et } \\
\text { al., } 2011\end{array}$ \\
\hline 7 & $\begin{array}{r}\text { Tephrosia } \\
\text { Purpure }\end{array}$ & $\begin{array}{r}\text { Whole } \\
\text { Plant }\end{array}$ & Aqueous & Streptozotocin & $\begin{array}{c}300 \text { and } \\
500 \mathrm{mg}\end{array}$ & $\begin{array}{c}\text { Shraddha V.et } \\
\text { al., } 2015\end{array}$ \\
\hline
\end{tabular}




\section{Cardioprotective Plant Extracts}

Table No IX: Extract of Other parts of plant for cardiomyopathy activity

\begin{tabular}{|c|c|c|c|c|c|c|}
\hline S. No & Plant Name & $\begin{array}{l}\text { Parts } \\
\text { Of } \\
\text { Plant }\end{array}$ & Extract & $\begin{array}{c}\mathrm{CMP} \\
\text { Induction }\end{array}$ & Concentration & Reference \\
\hline 1 & $\begin{array}{c}\text { Chonemorpha } \\
\text { fragrans }\end{array}$ & Root & Ethanol & Normal & $\begin{array}{c}100 \mathrm{mg} \text { and } \\
200 \mathrm{mg}\end{array}$ & $\begin{array}{c}\text { Mathew } \\
\text { George et al., } \\
2017\end{array}$ \\
\hline 2 & $\begin{array}{l}\text { Tinospora } \\
\text { cordifolia }\end{array}$ & Root & Alchol & Streptozotocin & $200 \mathrm{mg}$ & $\begin{array}{c}\text { Arshiya } \\
\text { Shamim et al., } \\
2015\end{array}$ \\
\hline 3 & $\begin{array}{c}\text { Newbouldia } \\
\text { laevis }\end{array}$ & $\begin{array}{l}\text { Leaf } \\
\text { and } \\
\text { Root }\end{array}$ & Water & $\begin{array}{c}\text { Carbon } \\
\text { Tetrachloride }\end{array}$ & $800 \mathrm{mg}$ & $\begin{array}{c}\text { K. N. Agbafor et } \\
\text { al., } 2015\end{array}$ \\
\hline 4 & $\begin{array}{l}\text { Marrubium } \\
\text { vulgaria } L .\end{array}$ & $\begin{array}{r}\text { Aerial } \\
\text { Part }\end{array}$ & Methanol & Isoproterenol & $40 \mathrm{mg}$ & $\begin{array}{c}\text { Keyvan Youshifi } \\
\text { et al., } \\
2013\end{array}$ \\
\hline 5 & Tecoma stans & Flower & Ethanol & Streptozotocin & $120 \mathrm{mg}$ & $\begin{array}{c}\text { Kameshwaran } \\
\text { Sugavanam et } \\
\text { al., } 2013\end{array}$ \\
\hline \multirow[t]{2}{*}{6} & Coconut Water & & & Doxorubicin & $3 \mathrm{ml}$ & Nnodim \\
\hline & & & & & & $\begin{array}{c}\text { Johnkennedy et } \\
\text { al., } 2013\end{array}$ \\
\hline 7 & $\begin{array}{c}\text { SolanumNigrum } \\
\text { Linn. }\end{array}$ & Berries & Methanol & Normal & 2.5 and $5 \mathrm{mg}$ & $\begin{array}{c}\text { Bhatia Nitish et } \\
\text { al., } 2011\end{array}$ \\
\hline 8 & $\begin{array}{c}\text { Moringa oleifera } \\
\text { Lam. }\end{array}$ & $\begin{array}{c}\text { Stem \& } \\
\text { Bark }\end{array}$ & Aqueous & Isoproterenol & $500 \mathrm{mg}$ & $\begin{array}{l}\text { Mahendra A } \\
\text { Gunjal et al., } \\
2010\end{array}$ \\
\hline 9 & Picrorhiza kurroa & Root & Ethanol & Adriamycin & $50 \mathrm{mg}$ & $\begin{array}{c}\text { D. } \\
\text { Rajaprabhu., et } \\
\text { al., } 2007\end{array}$ \\
\hline 10 & Date Palm & Pollen & Ethanol & isoproterenol & $400 \mathrm{mg}$ & $\begin{array}{l}\text { Amal Daoud et } \\
\text { al., } 2017\end{array}$ \\
\hline
\end{tabular}

\section{CONCLUSION}

The review reveal that the natural products as plant extract gives more protection against cardiomyopathy. From this we can clear that the cardiomyopathy were induced by many different inducing factors but it was treated mostly by medicinally important traditional plants only, because it doesn't cause very serious side effects or no side effects. For reversing heart diseases and also preventing diet and lifestyle plays an important role in our healthy life.

\section{REFERENCES}

1. S. Krisela, "The heart and stroke foundation South Africa heart disease in South Africa Media data document," ed, 2007.

2. J. Gowri, A. V. Anand, S. Achiraman, G. R. Archunan, and S. Kalavathy, "Redemptive benefit of atorvastatin in the risk factors of coronary artery disease," Int J Cur Biomed Phar Res., vol. 1, pp. 15-19, 2011.

3. C. Hennekens, "Clinical and research challenges in risk factors for cardiovascular diseases," European heart journal, vol. 21, pp. 1917-1921, 2000.
4. D. Rajaprabhu, R. Rajesh, R. Jeyakumar, S. Buddhan, B. Ganesan, and R. An, "Protective effect of Picrorhiza kurroa on antioxidant defense status in adriamycin-induced cardiomyopathy in rats," Journal of Medicinal Plants Research, vol. 1, pp. 080-085, 2007.

5. Kumar, G. Krishna, and P. Hullatti, "Indian Plants with Cardioprotective Activity-A Review," Systematic Reviews in Pharmacy, vol. 8, p. 8, 2017.

6. S. Parasuraman, G. S. Thing, and S. A. Dhanaraj, "Polyherbal formulation: Concept of ayurveda," Pharmacognosy reviews, vol. 8, p. 73, 2014.

7. N. Rao, "Bioactive phytochemicals in Indian foods and their potential in health promotion and disease prevention," Asia Pacific Journal of clinical nutrition, vol. 12, 2003.

8. U. Dhar, R. S. Rawal, S. S. Samant, S. Airi, and J. Upreti, "People's participation in Himalayan biodiversity conservation: a practical approach," Current Science, vol. 76, pp. 36-40, 1999.

9. Pattanaik, "Some phytotherapeutic claims by tribals of Rayagada district, Orissa, India," Ethnobotanical Leaflets, vol. 2006, p. 20, 2006.

10. G. B. Mahady, "Medicinal plants for the prevention and treatment of coronary heart disease," Ethnopharmacology-Volume II, p. 75, 2009.

11. R. A. Khan, "Protective effects of Sonchus asper (L.) Hill,(Asteraceae) against $\mathrm{CCl} 4$-induced oxidative stress in the thyroid tissue of rats," BMC complementary and alternative medicine, vol. 12, p. $181,2012$. 
12. SAH AND P. Nagarathana, "SCREENing OF CaRdioprotective ACTIVITY OF LEAVES OF ANDROGRAPHIS PANICULATA AgAINST ISOPROTERENOL INDUCED MYOCARDIAL INFARCTION IN RATS," INT. J. PHARMACOL. Res, Vol. 6, PP. 23-28, 2016.

13. E. H. Choi, H.-J. Chang, J. Y. Cho, and H. S. Chun, "Cytoprotective effect of anthocyanins against doxorubicin-induced toxicity in H9c2 cardiomyocytes in relation to their antioxidant activities," Foodand chemical toxicology, vol. 45, pp. 1873-1881, 2007.

14. Hiona, A. S. Lee, J. Nagendran, X. Xie, A. J. Connolly, R. C. Robbins, et al., "Pretreatment with angiotensin-converting enzyme inhibitor improves doxorubicininduced cardiomyopathy via preservation of mitochondrial function," The Journal of thoracic and cardiovascular surgery, vol. 142, pp. 396-403. e3, 2011.

15. Z. Wang and H. Gleichmann, "GLUT2 in pancreatic islets: crucial target molecule in diabetes induced with multiple low doses of streptozotocin in mice," Diabetes, vol. 47, pp. 50-56, 1998.

16. S. Panda and S. R. Naik, "Evaluation of cardioprotective activity of Ginkgo biloba and Ocimum sanctum in rodents," Alternative Medicine Review, vol. 14, p. 161, 2009.

\section{AUTHORS PROFILE}

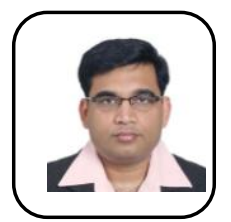

Dr. Sankarganash Arunachaalam, currently works at the Faculty of Bio-Technology, Kalasalingam Academy o Research and Education. He does research in Bioinformatics, Cancer Research and Cell Biology.

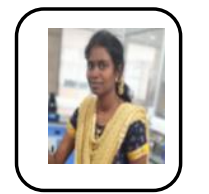

Umapriya. M, is a $\mathrm{PhD}$ Scholar in Kalasalingam Academy of Research and Education. She completed her UG and PG degree in Microbiology at ANJAC, Sivakasi. Her area of Research in Drug Toxicology and Adverse Drug Reactions.

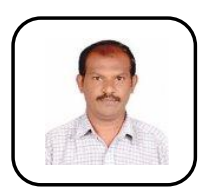

Dr. Selvaraj Kunjiappan currently works at the Department of Biotechnology, Kalasalingam University. Their current project is 'Design, Graph Theoretical Identification and Comparative Molecular Docking Analysis of Biologically Important Nanoparticles . 